\title{
On an Interpretation of Second Order Quantification in First Order Intuitionistic Propositional Logic
}

\author{
Andrew M. Pitts* \\ University of Cambridge Computer Laboratory \\ Cambridge CB2 3QG England \\ 〈Andrew.Pitts@cl.cam.ac.uk〉
}

\begin{abstract}
We prove the following surprising property of Heyting's intuitionistic propositional calculus, IpC. Consider the collection of formulas, $\phi$, built up from propositional variables $(p, q, r, \ldots)$ and falsity $(\perp)$ using conjunction $(\wedge)$, disjunction $(\vee)$ and implication $(\rightarrow)$. Write $\vdash \phi$ to indicate that such a formula is intuitionistically valid. We show that for each variable $p$ and formula $\phi$ there exists a formula $A_{p} \phi$ (effectively computable from $\phi$ ), containing only variables not equal to $p$ which occur in $\phi$, and such that for all formulas $\psi$ not involving $p, \vdash \psi \rightarrow A_{p} \phi$ if and only if $\vdash \psi \rightarrow \phi$. Consequently quantification over propositional variables can be modelled in IpC, and there is an interpretation of the second order propositional calculus, $\mathrm{IpC}^{2}$, in $\mathrm{IpC}$ which restricts to the identity on first order propositions.

An immediate corollary is the strengthening of the usual Interpolation Theorem for $\mathrm{IpC}$ to the statement that there are least and greatest interpolant formulas for any given pair of formulas. The result also has a number of interesting consequences for the algebraic counterpart of IpC, the theory of Heyting algebras. In particular we show that a model of $\mathrm{IpC}^{2}$ can be constructed whose algebra of truth-values is equal to any given Heyting algebra.
\end{abstract}

*Supported by the ESPRIT Basic Research Action Nr 3003, 'CLICS'. 


\section{Introduction}

This paper establishes a new and rather surprising property of Heyting's intuitionistic propositional calculus, IpC. We show that quantification over propositional variables can be modelled in $\mathrm{IpC}$, and hence that there is an interpretation of the second order propositional calculus, $\mathrm{IpC}^{2}$, in $\mathrm{IpC}$ which restricts to the identity on first order propositions. In order to state this result more precisely, we briefly recall the syntax and proof theory of first and second order intuitionistic propositional logic.

We will take the first order propositions, $\phi$, to be given by the following grammar

$$
\phi::=p|\perp| \phi \wedge \phi^{\prime}\left|\phi \vee \phi^{\prime}\right| \phi \rightarrow \phi^{\prime}
$$

where $p$ ranges over a set of propositional variables. Negation, truth and biimplication can be defined in the usual way:

$$
\neg \phi \stackrel{\text { def }}{=} \phi \rightarrow \perp \quad \top \stackrel{\text { def }}{=} \neg \perp \quad \phi \leftrightarrow \phi^{\prime} \stackrel{\text { def }}{=}\left(\phi \rightarrow \phi^{\prime}\right) \wedge\left(\phi^{\prime} \rightarrow \phi\right)
$$

Table 1 gives a collection of natural deduction style rules for IpC. The premisses and conclusion of each rule are sequents, $\Gamma \Rightarrow \phi$, which we take to be specified by a finite multiset (unordered list) of first order propositions, $\mathrm{T}$, and a single first order proposition $\phi$. The use of multisets rather than sets in sequents will be important when we consider the 'size' of sequents in section 2. For the moment, we note that since the order of formulas in $\Gamma$ is immaterial, an explicit structural rule of Exchange is not needed. As usual, we identify formulas with one-element multisets, and write $\Gamma \Delta$ for the union of two multisets $\Gamma$ and $\Delta$. We will write

$$
\mathrm{IpC} \vdash \mathrm{\Gamma} \Rightarrow \phi
$$

to indicate that the sequent is provable using the rules in Table 1 . The new property of IpC which we establish here is:

Theorem 1 Given a propositional variable $p$, for each first order proposition $\phi$ there is a first order proposition $A_{p} \phi$, containing only variables not equal to $p$ which occur in $\phi$, and satisfying:

(i) If $\mathrm{IpC} \vdash \Gamma \Rightarrow \phi$, then $\mathrm{IpC} \vdash \Gamma \Rightarrow A_{p} \phi$, provided $p$ does not occur in $\mathrm{\Gamma}$.

(ii) If $\mathrm{IpC} \vdash \Gamma \Rightarrow A_{p} \phi$, then for all $\psi$, IpC $\vdash \Gamma \Rightarrow \phi[\psi / p]$ (where $\phi[\psi / p]$ denotes the result of substituting $\psi$ for $p$ throughout $\phi)$.

This theorem will be proved in section 2 using proof-theoretic methods. The key tool is the use of a Gentzen-style sequent calculus for IpC for which there is a well-founded relation on sequents making the hypotheses of each rule of the calculus less than its conclusion. (The first order proposition $A_{p} \phi$ will be defined by recursion over this well-founded relation.) The particular sequent calculus 
we use is that given (independently) by Hudelmaier [6] and Dyckhoff [4]; its implicational part (the most important part) also occurs in the work of Lincoln, Scedrov and Shankar [8]. In fact, essentially similar refinements of the sequent calculus for IpC were developed by the Russian school of Proof Theory some time ago-see Vorob'ev [13].

Remark 2 It is perhaps worth pointing out that the analogue of Theorem 1 for classical logic is rather trivially true, since there we may take $A_{p} \phi$ to be $\phi[\top / p] \wedge \phi[\perp / p]$.

The existence of first order propositions $A_{p} \phi$ satisfying Theorem 1 enables one to interpret in $\mathrm{IpC}$ the second order intuitionistic propositional logic, $\mathrm{IpC}^{2}$, a logic which extends IpC with quantification over propositional variables. As is well known, in this logic implication and universal quantification suffice to define the other connectives and existential quantification-see [11]. However, we will take the second order propositions to be given by the grammar

$$
\phi::=p|\perp| \phi \wedge \phi^{\prime}\left|\phi \vee \phi^{\prime}\right| \phi \rightarrow \phi^{\prime} \mid \forall p \phi
$$

in order that they be a superset of the first order propositions. The natural deduction rules for $\forall p \phi$ are given in Table 2. Occurrences of $p$ in $\phi$ become bound in $\forall p \phi$; all other types of occurrence of variables are free. We will write

$$
\mathrm{IpC}^{2} \vdash \Gamma \Rightarrow \phi
$$

to indicate that a sequent of second order propositions can be proved using the rules in Tables 1 and 2 .

In section 3 we use Theorem 1 to define a translation of second order propositions, $\phi$, into first order ones, $\phi^{*}$. The translation has the following properties:

(i) If $\mathrm{IpC}^{2} \vdash \Gamma \Rightarrow \phi$, then $\mathrm{IpC} \vdash \Gamma^{*} \Rightarrow \phi^{*}$ (where $\Gamma^{*}$ indicates the translation applied elementwise to the multiset $\Gamma$ ).

(ii) $p^{*}=p, \perp^{*}=\perp,(\phi \# \psi)^{*}=\phi^{*} \# \psi^{*}$ (for $\#=\wedge, \vee, \rightarrow$ ), and hence in particular $\phi^{*}=\phi$ for all first order propositions $\phi$.

See Proposition 9 below for more details. An immediate corollary of the existence of such an interpretation is a strengthening of the usual Interpolation Theorem for IpC. Recall that the latter says that given first order propositions $\phi$ and $\psi$ for which $\phi \Rightarrow \psi$ is provable in $\mathrm{IpC}$, there is some first order proposition $\theta$ containing only variables common to both $\phi$ and $\psi$, and for which both $\phi \Rightarrow \theta$ and $\theta \Rightarrow \psi$ are provable. Here we establish (Proposition 11) that the collection of such interpolant propositions is not merely non-empty but in fact contains least and greatest elements (with respect to the provability ordering for $\mathrm{IpC}{ }^{1}$

\footnotetext{
${ }^{1}$ The author is grateful to G. R. Renardel de Lavalette for pointing out that this solves his open problem 6.5 in $[\mathrm{RdL}]$.
} 


$$
\begin{aligned}
& \frac{\Gamma \Rightarrow \psi}{\Gamma \phi \Rightarrow \psi}(\text { Weaken }) \quad \frac{\Gamma \phi \phi \Rightarrow \psi}{\Gamma \phi \Rightarrow \psi}(\text { Contract }) \\
& \overline{\phi \Rightarrow \phi}(I d) \\
& \frac{\Gamma \Rightarrow \perp}{\Gamma \Rightarrow \phi}(\perp \text { Elim }) \\
& \frac{\Gamma \Rightarrow \phi \Delta \Rightarrow \psi}{\Gamma \Delta \Rightarrow \phi \wedge \psi}(\wedge \text { Intr }) \quad \frac{\Gamma \Rightarrow \phi \wedge \psi}{\Gamma \Rightarrow \phi}\left(\wedge \text { Elim }_{1}\right) \quad \frac{\Gamma \Rightarrow \phi \wedge \psi}{\Gamma \Rightarrow \psi}\left(\wedge \text { Elim }_{2}\right) \\
& \frac{\Gamma \Rightarrow \phi}{\Gamma \Rightarrow \phi \vee \psi}\left(\vee \operatorname{Intr}_{1}\right) \quad \frac{\Gamma \Rightarrow \psi}{\Gamma \Rightarrow \phi \vee \psi}\left(\vee \operatorname{Intr}_{2}\right) \\
& \frac{\Gamma \phi \Rightarrow \theta \quad \Delta \psi \Rightarrow \theta \quad \Theta \Rightarrow \phi \vee \psi}{\Gamma \Delta \Theta \Rightarrow \theta}(\text { Elim }) \\
& \frac{\Gamma \phi \Rightarrow \psi}{\Gamma \Rightarrow \phi \rightarrow \psi}(\rightarrow \text { Intr }) \quad \frac{\Gamma \Rightarrow \phi \rightarrow \psi \quad \Delta \Rightarrow \phi}{\Gamma \Delta \Rightarrow \psi}(\rightarrow \text { Elim })
\end{aligned}
$$

Table 1: Natural deduction rules for IpC

$$
\frac{\Gamma \Rightarrow \phi}{\Gamma \Rightarrow \forall p \phi}(\forall \text { Intr })^{*} \quad \frac{\Gamma \Rightarrow \forall p \phi}{\Gamma \Rightarrow \phi[\psi / p]}(\forall \text { Elim })
$$

$\left({ }^{*}\right.$ provided $p$ is not free in $\Gamma$ )

Table 2: Natural deduction rules for $\forall$ 
Theorem 1 also has interesting consequences for the theory of Heyting algebras, a theory which bears the same relationship to $\mathrm{IpC}$ as does the theory of Boolean algebras to classical propositional logic. In section 4 we establish a form of 'second order completeness' for Heyting algebras: letting $H[X]$ denote the Heyting algebra obtained by adjoining an indeterminate $X$ to a given Heyting algebra $H$, we prove (Theorem 13) that the inclusion

$$
i_{H}: H \hookrightarrow H[X]
$$

possesses both left and right adjoints, i.e. there are monotone functions, $a_{H}, e_{H}$ : $H[X] \rightarrow H$, satisfying $\left(e_{H} \circ i_{H}\right)=i d_{H}=\left(a_{H} \circ i_{H}\right)$ and $\left(i_{H} \circ a_{H}\right) \leq i d_{H[X]} \leq$ $\left(i_{H} \circ e_{H}\right)$. Moreover, these adjoints are natural in $H$ in the category-theoretic sense. It follows from this that given any Heyting algebra $H$, the generic model of the algebraic theory of 'Heyting algebras equipped with a morphism from $H$ ' is actually a model of $\mathrm{IpC}^{2}$. Since the algebra of truth-values of this model is just $H$, we conclude (Proposition 18) that any Heyting algebra can appear as the algebra of truth-values of a model of $\mathrm{IpC}^{2}$. Equivalently, any Heyting algebra can appear as the Lindenbaum algebra of a second order intuitionistic theory.

The results presented in this paper have had a rather long gestation period. Some ten or so years ago I tried to prove the negation of Theorem 1 in connection with the higher order analogue of Proposition 18-the question whether any Heyting algebra can appear as the algebra of truth-values of an elementary topos. I established that the free Heyting algebra on a countable infinity of generators does not so appear provided the property of $\mathrm{IpC}$ given in Theorem 1 does not hold. It seemed likely to me (and to others to whom I posed the question) that a first order proposition $\phi$ could be found for which $A_{p} \phi$ does not exist (although I could not find one!), thus settling the original question about toposes and Heyting algebras in the negative. That Theorem 1 is true is quite a surprise to me. Unfortunately, it appears that not all the results for second order logic reported here generalize to the setting of higher order logic. Whilst it is the case that Theorem 1 remains true if $\mathrm{IpC}$ is replaced by a quantifier-free fragment of intuitionistic higher order logic, the substitution property of Lemma 8 fails (so that one does not get an interpretation of full higher order logic in its quantifierfree fragment). It remains an open question whether every Heyting algebra can be the Lindenbaum algebra of a theory in intuitionistic higher order logic.

Acknowledgement I would like to thank R. Dyckhoff for bringing to my attention the particular sequent calculus for IpC used in this paper. I would also like to thank him, A. Scedrov and G. Mints for elucidating its history. 


$$
\begin{gathered}
\frac{\Gamma p p}{\Gamma p \Rightarrow \text { Atom })^{*}} \\
\frac{\Gamma \perp \phi}{\Gamma \Rightarrow}(\perp) \\
\frac{\Gamma \Rightarrow \phi \quad \Gamma \Rightarrow \psi}{\Gamma \Rightarrow \phi \wedge \psi}(\Rightarrow \wedge) \quad \frac{\Gamma \phi \psi \Rightarrow \theta}{\Gamma(\phi \wedge \psi) \Rightarrow \theta}(\wedge \Rightarrow) \\
\frac{\Gamma \Rightarrow \psi}{\Gamma \Rightarrow \phi \vee \psi}\left(\Rightarrow \vee_{1}\right) \quad \frac{\Gamma \phi \Rightarrow \theta \quad \Gamma \psi \Rightarrow \theta}{\Gamma(\phi \vee \psi) \Rightarrow \theta}(\vee \Rightarrow) \\
\frac{\Gamma \phi \Rightarrow \psi}{\Gamma \Rightarrow \phi \rightarrow \psi}(\Rightarrow \rightarrow) \quad \frac{\Gamma(\phi \rightarrow \psi) \Rightarrow \phi \quad \Gamma \psi \Rightarrow \theta}{\Gamma(\phi \rightarrow \psi) \Rightarrow \theta}(\rightarrow \Rightarrow) \\
\left({ }^{*} \text { where } p \text { is any propositional variable }\right)
\end{gathered}
$$

Table 3: Sequent calculus for IpC

\section{Proof of Theorem 1}

Our proof will use the methods of Proof Theory. To be more precise, we will employ a certain refinement of the cut-free Gentzen sequent calculus for IpC and we begin by explaining that.

Table 3 contains a fairly standard cut-free sequent calculus for IpC. This formulation of the sequent calculus has essential uses of the structural rules (Weaken) and (Contract) of Table 1 built in implicitly: weakening is built in via the (Atom) axiom, and an essential use of contraction is built in to the rule $(\rightarrow \Rightarrow)$ by repeating the active proposition $\phi \rightarrow \psi$ in the first premiss of the rule. We refer the reader to $[3$, Part $1, \S 3]$ for a proof of the fact that (Weaken), (Contract), (Id) and the Cut Rule

$$
\frac{\Gamma \Rightarrow \phi \quad \phi \Delta \Rightarrow \psi}{\Gamma \Delta \Rightarrow \psi}(C u t)
$$

are all derivable from the rules in Table 3 , and hence that these rules determine the same provable sequents as do those in Table 1.

Note that $(\rightarrow \Rightarrow)$ is the only rule in Table 3 which fails to have the property that its premisses are structurally simpler than its conclusion. Following Dyckhoff [4] and Hudelmaier [6], we can overcome this defect by replacing $(\rightarrow \Rightarrow)$ by 


$$
\begin{gathered}
\frac{\Gamma p \phi \Rightarrow \psi}{\Gamma p(p \rightarrow \phi) \Rightarrow \psi}\left(\rightarrow \Rightarrow_{1}\right)^{*} \\
\frac{\Gamma\left(\phi_{1} \rightarrow\left(\phi_{2} \rightarrow \phi_{3}\right)\right) \Rightarrow \psi}{\Gamma\left(\left(\phi_{1} \wedge \phi_{2}\right) \rightarrow \phi_{3}\right) \Rightarrow \psi}\left(\rightarrow \Rightarrow_{2}\right) \\
\frac{\Gamma\left(\phi_{1} \rightarrow \phi_{3}\right)\left(\phi_{2} \rightarrow \phi_{3}\right) \Rightarrow \psi}{\Gamma\left(\left(\phi_{1} \vee \phi_{2}\right) \rightarrow \phi_{3}\right) \Rightarrow \psi}\left(\rightarrow \Rightarrow_{3}\right) \\
\frac{\Gamma\left(\phi_{2} \rightarrow \phi_{3}\right) \Rightarrow \phi_{1} \rightarrow \phi_{2} \quad \Gamma \phi_{3} \Rightarrow \psi}{\Gamma\left(\left(\phi_{1} \rightarrow \phi_{2}\right) \rightarrow \phi_{3}\right) \Rightarrow \psi}(\rightarrow \Rightarrow 4) \\
\left({ }^{*} \text { where } p \text { is any propositional variable }\right) \\
\text { Table 4: Replacements for }(\rightarrow \Rightarrow)
\end{gathered}
$$

the four rules shown in Table 4. The new rules correspond to the possible forms of the antecedent of the introduced implication-except that a separate rule is not needed for the case when the antecedent is $\perp$ (since the appropriate rule is an instance of weakening).

We will refer to the system of rules obtained from Table 3 by relacing $(\rightarrow \Rightarrow)$ by the rules in Table 4 as $\mathrm{LJ}^{*}$. Clearly any sequent provable in L.J is intuitionistically valid, since the rules in Table 4 are all derivable in $\mathrm{IpC}_{\mathrm{p}}$; the converse is also true, so that one has:

Theorem $3 \mathrm{IpC} \vdash \mathrm{\Gamma} \Rightarrow \phi$ if and only if the sequent is provable in $\mathrm{LJ}^{*}$

We refer the reader to [4, Theorem 1] for a proof of this result.

Definition 4 The weight, wt $(\phi)$, of a first order proposition $\phi$ is a positive integer defined by induction on the structure of $\phi$ as follows:

$$
\begin{gathered}
w t(p)=w t(\perp)=1 \\
w t(\phi \vee \psi)=w t(\phi \rightarrow \psi)=w t(\phi)+w t(\psi)+1 \\
w t(\phi \wedge \psi)=w t(\phi)+w t(\psi)+2
\end{gathered}
$$

This weight function defines a well-ordering, $\prec$, on first order propositions via the definition

$$
\phi \prec \psi \quad \text { if and only if } w t(\phi)<w t(\psi)
$$


Now extend $\prec$ to a relation between finite multisets of propositions via the multiset ordering construction of Dershowitz and Manna. Thus

$$
\Gamma \prec \Delta
$$

holds if and only if $\Delta=\Delta_{1} \Delta_{2}$ and $\Gamma=\Delta_{1} \Gamma_{2}$, for some $\Delta_{1}, \Delta_{2}$ and $\Gamma_{2}$ with $\Delta_{2}$ non-empty and such that for all $\gamma \in \Gamma_{2}$ there exists $\delta \in \Delta_{2}$ with $\gamma \prec \delta$. As shown in [2], this relation between multisets is well-founded because the original relation on propositions is.

Finally, define a well-founded relation on sequents by declaring

$$
(\Gamma \Rightarrow \phi) \prec(\Delta \Rightarrow \psi)
$$

to hold just in case $\Gamma \phi \prec \Delta \psi$. Note that each rule in L.J* has the property that a (premiss,conclusion)-pair lies in this relation between sequents.

We turn now to the proof of Theorem 1. Recall that in second order intuitionistic propositional logic, existentially quantified propositions, $\exists p \phi$, are definable in terms of $\forall$ and $\rightarrow$ :

$$
\exists p \phi \stackrel{\text { def }}{=} \forall q(\forall p(\phi \rightarrow q) \rightarrow q)
$$

(where $q$ is not free in $\phi$ ). It follows that the existence of the first order propositions $A_{p} \phi$ for all $\phi$, with properties as in Theorem 1, entails the existence of first order propositions $E_{p} \phi$ which model the existentially quantified proposition $\exists p \phi$. In fact to prove the theorem, we will need to define $A_{p} \phi$ and $E_{p} \phi$ simultaneously via mutual recursion. Moreover, we will need to give the definitions for multisets of formulas rather than for single formulas $\phi$, in order to utilize $\mathrm{LJ}^{*}$ to prove the required properties of the construction.

Proposition 5 Let $\Delta$ be a finite multiset of first order propositions and $\phi$ a single first order proposition. For each variable $p$ there are first order propositions

$$
E_{p}(\Delta) \quad \text { and } \quad A_{p}(\Delta ; \phi)
$$

satisfying:

(i) (a) $\operatorname{Var}\left(E_{p}(\Delta)\right) \subseteq \operatorname{Var}(\Delta) \backslash\{p\}$

(b) $\operatorname{Var}\left(A_{p}(\Delta ; \phi)\right) \subseteq \operatorname{Var}(\Delta \phi) \backslash\{p\}$

where $\operatorname{Var}(\Delta)$ denotes the finite set of propositional variables in $\Delta$.

(ii) (a) $\operatorname{IpC} \vdash \Delta \Rightarrow E_{p}(\Delta)$

(b) $\mathrm{IpC} \vdash \Delta A_{p}(\Delta ; \phi) \Rightarrow \phi$

Moreover, for all finite multisets of first order propositions $\Gamma$ not containing $p$, if $\mathrm{IpC} \vdash \Gamma \Delta \Rightarrow \phi$, then: 
(iii) (a) $\operatorname{IpC} \vdash \Gamma E_{p}(\Delta) \Rightarrow \phi$, provided $p \notin \operatorname{Var}(\phi)$

(b) $\operatorname{IpC} \vdash \Gamma E_{p}(\Delta) \Rightarrow A_{p}(\Delta ; \phi)$

Theorem 1 follows immediately from Proposition 5 if we make the definition

$$
A_{p} \phi \stackrel{\text { def }}{=} A_{p}(\emptyset ; \phi)
$$

For if $\mathrm{IpC} \vdash \Gamma \Rightarrow \phi$ with $p \notin \operatorname{Var}(\Gamma)$, then by 5 (iii)(b)

$$
\mathrm{IpC} \vdash \Gamma E_{p}(\emptyset) \Rightarrow A_{p}(\emptyset ; \phi)
$$

and by $5(\mathrm{ii})(\mathrm{a})$

$$
\mathrm{IpC} \vdash \emptyset \Rightarrow E_{p}(\emptyset)
$$

which together give IpC $\vdash \Gamma \Rightarrow A_{p} \phi$, as required for part (i) of the theorem. For part (ii) of the theorem, note that 5 (ii)(b) gives

$$
\mathrm{IpC} \vdash A_{p} \phi \Rightarrow \phi
$$

Hence the sequent obtained from this one by substituting a proposition $\psi$ for $p$ throughout, is also provable; but by $5(\mathrm{i})(\mathrm{b}) p \notin \operatorname{Var}\left(A_{p} \phi\right)$, so the result of such a substitution is $\mathrm{IpC} \vdash A_{p} \phi \Rightarrow \phi[\psi / p]$. So $\operatorname{IpC} \vdash \Gamma \Rightarrow \phi[\psi / p]$ holds whenever $\mathrm{IpC} \vdash \Gamma \Rightarrow A_{p} \phi$ does, as required.

Remark 6 Properties (i)(a), (ii)(a) and (iii)(a) together imply that $E_{p}(\Delta)$ acts like the existentially quantified formula $\exists p(\wedge \Delta)$ (where $\wedge \Delta$ denotes the conjunction of the formulas in $\Delta$ ). However, properties (i)(b), (ii)(a), (ii)(b) and (iii)(b) imply that it is $E_{p}(\Delta) \rightarrow A_{p}(\Delta ; \phi)$, rather than just $A_{p}(\Delta ; \phi)$, which acts like the universally quantified formula $\forall p(\wedge \Delta \rightarrow \phi)$. This is because of the appearance of $E_{p}(\Delta)$ in (iii)(b) - a complication which is needed to carry through the proof of (iii). This proof proceeds by induction on the structure of the proof of $\Gamma \Delta \Rightarrow \phi$ in $L J^{*}$, and it is the case where the proof ends with the active formula an implication contained in $\Gamma$ which requires us to prove (iii)(b) rather than $\mathrm{IpC} \vdash \Gamma \Rightarrow A_{p}(\Delta ; \phi)$.

The rest of this section will be devoted to the proof of Proposition 5.

The formulas $E_{p}(\Delta)$ and $A_{p}(\Delta ; \phi)$ are defined simultaneously by $\prec$-induction on $\Delta \phi$ (where $\prec$ is the well-founded relation of Definition 4 ). At each stage, we define $E_{p}(\Delta)$ as the conjunction of a finite set of formulas $\mathcal{E}_{p}(\Delta)$ and $A_{p}(\Delta ; \phi)$ as the disjunction of a finite set of formulas $\mathcal{A}_{p}(\Delta ; \phi)$ :

$$
\begin{aligned}
E_{p}(\Delta) & \stackrel{\text { def }}{=} \wedge \mathcal{E}_{p}(\Delta) \\
A_{p}(\Delta ; \phi) & \stackrel{\text { def }}{=} \vee \mathcal{A}_{p}(\Delta ; \phi)
\end{aligned}
$$


The elements of the finite sets $\mathcal{E}_{p}(\Delta)$ and $\mathcal{A}_{p}(\Delta ; \phi)$ are given by Table 5 , with one element for each match of $\Delta$ and $\Delta ; \phi$ to the patterns listed in the left-hand column of the table. It is quite possible that in a particular case there are no matches, so that $\mathcal{E}_{p}(\Delta)$ or $\mathcal{A}_{p}(\Delta ; \phi)$ is empty -in which case $E_{p}(\Delta)=\wedge \emptyset \stackrel{\text { def }}{=} \top$ and $A_{p}(\Delta ; \phi)=\vee \emptyset \stackrel{\text { def }}{=} \perp$.

It follows easily by $\prec$-induction on $\Delta \phi$ that $E_{p}(\Delta)$ and $A_{p}(\Delta ; \phi)$ are built up from subformulas of $\Delta \phi$ and that they do not contain $p$. So 5 (i) holds.

The validity of the sequents in 5 (ii) is proved simultaneously by $\prec$-induction on $\Delta \phi$. At each stage we have to show that

$$
\mathrm{IpC} \vdash \Delta \Rightarrow \varepsilon \quad \text { and } \quad \mathrm{IpC} \vdash \Delta \alpha \Rightarrow \phi
$$

hold for each $\varepsilon \in \mathcal{E}_{p}(\Delta)$ and each $\alpha \in \mathcal{A}_{p}(\Delta ; \phi)$. For each of the cases (E0)(E8) and (A1)-(A13) of Table 5, this follows from the induction hypothesis by straightforward proofs in IpC.

Turning now to 5 (iii), this is proved by induction on the structure of a proof of $\mathrm{T} \Delta \Rightarrow \phi$ in $\mathrm{LJ}^{*}$, with one case for each proof rule:

Case (Atom): So $\phi$ is a propositional variable and is an element of $\Gamma \Delta$, i.e. $\phi \in \Gamma$ or $\phi \in \Delta$. We will split the argument into two subcases according to whether $\phi$ is the variable $p$ or not.

Subcase $\phi=p$ : In this case we just have to check that (b) holds for $\Gamma \Delta \Rightarrow \phi$. Since $p \notin \Gamma$, we must have $\Delta=\Delta^{\prime} p$. Then case (A10) of Table 5 gives IpC $\vdash \top \Rightarrow A_{p}\left(\Delta^{\prime} p ; p\right)$, from which (b) follows.

Subcase $\phi \neq p$ : We know that either $\phi \in \Gamma$ or $\phi \in \Delta$. In the first case (a) holds for $\Gamma \Delta \Rightarrow \phi$ by (Atom), and (b) follows from (a) because by case (A9) of Table 5 , IpC $\vdash \phi \Rightarrow A_{p}(\Delta ; \phi)$. On the other hand, if $\phi \in \Delta$, say $\Delta=\Delta^{\prime} \phi$, case (E1) of Table 5 gives IpC $\vdash E_{p}\left(\Delta^{\prime} \phi\right) \Rightarrow E_{p}\left(\Delta^{\prime}\right) \wedge \phi$, from which (a) for $\Gamma \Delta \Rightarrow \phi$ follows; and as before (b) follows from (a) by case (A9).

Case $(\perp \Rightarrow)$ : So $\perp \in \Gamma \Delta$. If $\perp \in \Gamma$ then (a) and (b) hold by ( $\perp \Rightarrow)$. If $\perp \in \Delta$, then by case (E0) of Table $5 \vdash E_{p}(\Delta) \Rightarrow \perp$ from which (a) and (b) follow.

Case $(\Rightarrow \wedge)$ : So $\phi=\phi_{1} \wedge \phi_{2}, \vdash \Gamma \Delta \Rightarrow \phi_{i}(i=1,2)$ and (a) and (b) hold for these sequents by induction hypothesis.

(a) If $p \notin \operatorname{Var}(\phi)$ then $p \notin \operatorname{Var}\left(\phi_{i}\right)$, so by (a) for $\Gamma \Delta \Rightarrow \phi_{i}$ we have

$$
\operatorname{IpC} \vdash \Gamma E_{p}(\Delta) \Rightarrow \phi_{i}
$$

for $i=1,2$, from which (a) for $\Gamma \Delta \Rightarrow \phi$ follows. 


\begin{tabular}{|c|c|c|}
\hline & $\Delta$ matches & $\mathcal{E}_{p}(\Delta)$ contains \\
\hline$(E 0)$ & $\Delta^{\prime} \perp$ & $\perp$ \\
\hline$(E 1)$ & $\Delta^{\prime} q$ & $E_{p}\left(\Delta^{\prime}\right) \wedge q$ \\
\hline$(E 2)$ & $\Delta^{\prime}\left(\delta_{1} \wedge \delta_{2}\right)$ & $E_{p}\left(\Delta^{\prime} \delta_{1} \delta_{2}\right)$ \\
\hline$(E 3)$ & $\Delta^{\prime}\left(\delta_{1} \vee \delta_{2}\right)$ & $E_{p}\left(\Delta^{\prime} \delta_{1}\right) \vee E_{p}\left(\Delta^{\prime} \delta_{2}\right)$ \\
\hline$(E 4)$ & $\Delta^{\prime}(q \rightarrow \delta)$ & $q \rightarrow E_{p}\left(\Delta^{\prime} \delta\right)$ \\
\hline$(E 5)$ & $\Delta^{\prime} p(p \rightarrow \delta)$ & $E_{p}\left(\Delta^{\prime} p \delta\right)$ \\
\hline$(E 6)$ & $\Delta^{\prime}\left(\left(\delta_{1} \wedge \delta_{2}\right) \rightarrow \delta_{3}\right)$ & $E_{p}\left(\Delta^{\prime}\left(\delta_{1} \rightarrow\left(\delta_{2} \rightarrow \delta_{3}\right)\right)\right)$ \\
\hline$(E 7)$ & $\Delta^{\prime}\left(\left(\delta_{1} \vee \delta_{2}\right) \rightarrow \delta_{3}\right)$ & $E_{p}\left(\Delta^{\prime}\left(\delta_{1} \rightarrow \delta_{3}\right)\left(\delta_{2} \rightarrow \delta_{3}\right)\right)$ \\
\hline$(E 8)$ & $\Delta^{\prime}\left(\left(\delta_{1} \rightarrow \delta_{2}\right) \rightarrow \delta_{3}\right)$ & $\begin{array}{l}{\left[E_{p}\left(\Delta^{\prime}\left(\delta_{2} \rightarrow \delta_{3}\right)\right) \rightarrow A_{p}\left(\Delta^{\prime}\left(\delta_{2} \rightarrow \delta_{3}\right) ; \delta_{1} \rightarrow \delta_{2}\right)\right]} \\
\rightarrow E_{p}\left(\Delta^{\prime} \delta_{3}\right)\end{array}$ \\
\hline & $\Delta ; \phi$ matches & $\mathcal{A}_{p}(\Delta ; \phi)$ contains \\
\hline$(A 1)$ & $\Delta^{\prime} q ; \phi$ & $A_{p}\left(\Delta^{\prime} ; \phi\right)$ \\
\hline$(A 2)$ & $\Delta^{\prime}\left(\delta_{1} \wedge \delta_{2}\right) ; \phi$ & $A_{p}\left(\Delta^{\prime} \delta_{1} \delta_{2} ; \phi\right)$ \\
\hline$(A 3)$ & $\Delta^{\prime}\left(\delta_{1} \vee \delta_{2}\right) ; \phi$ & $\begin{array}{l}{\left[E_{p}\left(\Delta^{\prime} \delta_{1}\right) \rightarrow A_{p}\left(\Delta^{\prime} \delta_{1} ; \phi\right)\right]} \\
\quad \wedge\left[E_{p}\left(\Delta^{\prime} \delta_{2}\right) \rightarrow A_{p}\left(\Delta^{\prime} \delta_{2} ; \phi\right)\right]\end{array}$ \\
\hline$(A 4)$ & $\Delta^{\prime}(q \rightarrow \delta) ; \phi$ & $q \wedge A_{p}\left(\Delta^{\prime} \delta ; \phi\right)$ \\
\hline$(A 5)$ & $\Delta^{\prime} p(p \rightarrow \delta) ; \phi$ & $A_{p}\left(\Delta^{\prime} p \delta ; \phi\right)$ \\
\hline$(A 6)$ & $\Delta^{\prime}\left(\left(\delta_{1} \wedge \delta_{2}\right) \rightarrow \delta_{3}\right) ; \phi$ & $A_{p}\left(\Delta^{\prime}\left(\delta_{1} \rightarrow\left(\delta_{2} \rightarrow \delta_{3}\right)\right) ; \phi\right)$ \\
\hline$(A 7)$ & $\Delta^{\prime}\left(\left(\delta_{1} \vee \delta_{2}\right) \rightarrow \delta_{3}\right) ; \phi$ & $A_{p}\left(\Delta^{\prime}\left(\delta_{1} \rightarrow \delta_{3}\right)\left(\delta_{2} \rightarrow \delta_{3}\right) ; \phi\right)$ \\
\hline$(A 8)$ & $\Delta^{\prime}\left(\left(\delta_{1} \rightarrow \delta_{2}\right) \rightarrow \delta_{3}\right) ; \phi$ & $\begin{array}{l}{\left[E_{p}\left(\Delta^{\prime}\left(\delta_{2} \rightarrow \delta_{3}\right)\right) \rightarrow A_{p}\left(\Delta^{\prime}\left(\delta_{2} \rightarrow \delta_{3}\right) ; \delta_{1} \rightarrow \delta_{2}\right)\right]} \\
\wedge A_{p}\left(\Delta^{\prime} \delta_{3} ; \phi\right)\end{array}$ \\
\hline$(A 9)$ & $\Delta ; q$ & $q$ \\
\hline$(A 10)$ & $\Delta^{\prime} p ; p$ & $\top$ \\
\hline$(A 11)$ & $\Delta ; \phi_{1} \wedge \phi_{2}$ & $A_{p}\left(\Delta ; \phi_{1}\right) \wedge A_{p}\left(\Delta ; \phi_{2}\right)$ \\
\hline$(A 12)$ & $\Delta ; \phi_{1} \vee \phi_{2}$ & $A_{p}\left(\Delta ; \phi_{1}\right) \vee A_{p}\left(\Delta ; \phi_{2}\right)$ \\
\hline$(A 13)$ & $\Delta ; \phi_{1} \rightarrow \phi_{2}$ & $E_{p}\left(\Delta \phi_{1}\right) \rightarrow A_{p}\left(\Delta \phi_{1} ; \phi_{2}\right)$ \\
\hline
\end{tabular}

( $q$ denotes any propositional variable not equal to $p$ )

Table 5: Definition of $E_{p}(\Delta)$ and $A_{p}(\Delta ; \phi)$ 
(b) By (b) for $\Gamma \Delta \Rightarrow \phi_{i}$ we have

$$
\vdash \Gamma E_{p}(\Delta) \Rightarrow A_{p}\left(\Delta ; \phi_{i}\right)
$$

for $i=1,2$ and hence

$$
\mathrm{IpC} \vdash \Gamma E_{p}(\Delta) \Rightarrow A_{p}\left(\Delta ; \phi_{1}\right) \wedge A_{p}\left(\Delta ; \phi_{2}\right)
$$

But by case (A11) of Table 5

$$
\mathrm{IpC} \vdash A_{p}\left(\Delta ; \phi_{1}\right) \wedge A_{p}\left(\Delta ; \phi_{2}\right) \Rightarrow A_{p}(\Delta ; \phi)
$$

So (b) for $\Gamma \Delta \Rightarrow \phi$ follows.

Case $(\wedge \Rightarrow)$ :

Subcase: $\Gamma=\Gamma^{\prime}\left(\gamma_{1} \wedge \gamma_{2}\right)$, IpC $\vdash \Gamma^{\prime} \gamma_{1} \gamma_{2} \Delta \Rightarrow \phi$, and (a) and (b) hold for this sequent by induction hypothesis. Hence (a) and (b) hold for $\Gamma \Delta \Rightarrow \phi$ by an application of $(\wedge \Rightarrow)$.

Subcase: $\Delta=\Delta^{\prime}\left(\delta_{1} \wedge \delta_{2}\right)$, IpC $\vdash \Gamma \Delta^{\prime} \delta_{1} \delta_{2} \Rightarrow \phi$, and (a) and (b) hold for this sequent by induction hypothesis. From this it follows that (a) and (b) hold for $\mathrm{\Gamma} \Delta \Rightarrow \phi$ since by cases (E2) and (A2) of Table 5 we have

$$
\begin{aligned}
& \mathrm{IpC} \vdash E_{p}(\Delta) \Rightarrow E_{p}\left(\Delta^{\prime} \delta_{1} \delta_{2}\right) \\
& \mathrm{IpC} \vdash A_{p}\left(\Delta^{\prime} \delta_{1} \delta_{2} ; \phi\right) \Rightarrow A_{p}(\Delta ; \phi)
\end{aligned}
$$

Case $\left(\Rightarrow \vee_{i}\right)$ : This case is analagous to that for $(\Rightarrow \wedge)$, using case (A12) of Table 5 .

Case $(\vee \Rightarrow)$ :

Subcase: $\Gamma=\Gamma^{\prime}\left(\gamma_{1} \vee \gamma_{2}\right)$, IpC $\vdash \Gamma^{\prime} \gamma_{i} \Delta \Rightarrow \phi(i=1,2)$ and (a) and (b) hold for these sequents by induction hypothesis. Hence (a) and (b) hold for $\Gamma \Delta \Rightarrow \phi$ by an application of $(\vee \Rightarrow)$.

Subcase: $\Delta=\Delta^{\prime}\left(\delta_{1} \vee \delta_{2}\right)$, IpC $\vdash \Gamma \Delta^{\prime} \delta_{i} \Rightarrow \phi(i=1,2)$ and (a) and (b) hold for these sequents by induction hypothesis.

(a) If $p \notin \operatorname{Var}(\phi)$, then by (a) for $\Gamma \Delta^{\prime} \delta_{i} \Rightarrow \phi$ we have

$$
\mathrm{IpC} \vdash \Gamma E_{p}\left(\Delta^{\prime} \delta_{i}\right) \Rightarrow \phi
$$

for $i=1,2$, and thus

$$
\mathrm{IpC} \vdash \Gamma\left(E_{p}\left(\Delta^{\prime} \delta_{1}\right) \vee E_{p}\left(\Delta^{\prime} \delta_{1}\right)\right) \Rightarrow \phi
$$

But by case (E3) of Table 5

$$
\mathrm{IpC} \vdash E_{p}(\Delta) \Rightarrow E_{p}\left(\Delta^{\prime} \delta_{1}\right) \vee E_{p}\left(\Delta^{\prime} \delta_{1}\right)
$$

and hence (a) holds for $\Gamma \Delta \Rightarrow \phi$. 
(b) By (b) for $\Gamma \Delta^{\prime} \delta_{i} \Rightarrow \phi$ we have

$$
\mathrm{IpC} \vdash \Gamma E_{p}\left(\Delta^{\prime} \delta_{i}\right) \Rightarrow A_{p}\left(\Delta^{\prime} \delta_{i} ; \phi\right)
$$

for $i=1,2$, and thus

$$
\mathrm{IpC} \vdash \Gamma \Rightarrow\left(E_{p}\left(\Delta^{\prime} \delta_{1}\right) \rightarrow A_{p}\left(\Delta^{\prime} \delta_{1} ; \phi\right)\right) \wedge\left(E_{p}\left(\Delta^{\prime} \delta_{2}\right) \rightarrow A_{p}\left(\Delta^{\prime} \delta_{2} ; \phi\right)\right)
$$

But by case (A3) of Table 5

$$
\begin{aligned}
\mathrm{IpC} \vdash & \left(E_{p}\left(\Delta^{\prime} \delta_{1}\right) \rightarrow A_{p}\left(\Delta^{\prime} \delta_{1} ; \phi\right)\right) \wedge\left(E_{p}\left(\Delta^{\prime} \delta_{2}\right) \rightarrow A_{p}\left(\Delta^{\prime} \delta_{2} ; \phi\right)\right) \\
& \Rightarrow A_{p}(\Delta ; \phi)
\end{aligned}
$$

so that $\mathrm{IpC} \vdash \Gamma \Rightarrow A_{p}(\Delta ; \phi)$, and hence in particular (b) holds for $\Gamma \Delta \Rightarrow \phi$.

Case $(\Rightarrow \rightarrow)$ : So $\phi=\phi_{1} \rightarrow \phi_{2}$, IpC $\vdash \Gamma \Delta \phi_{1} \Rightarrow \phi_{2}$ and (a) and (b) hold for this sequent by induction hypothesis.

(a) If $p \notin \operatorname{Var}(\phi)$ then $p \notin \operatorname{Var}\left(\phi_{i}\right)$ for $i=1,2$. So by (a) for $\Gamma \Delta \phi_{1} \Rightarrow \phi_{2}$ we have

$$
\mathrm{IpC} \vdash \Gamma \phi_{1} E_{p}(\Delta) \Rightarrow \phi_{2}
$$

and hence by an application of $(\Rightarrow \rightarrow)$, (a) holds for $\Gamma \Delta \Rightarrow \phi$.

(b) By (b) for $\Gamma \Delta \phi_{1} \Rightarrow \phi_{2}$ we have

$$
\mathrm{IpC} \vdash \mathrm{T} E_{p}\left(\Delta \phi_{1}\right) \Rightarrow A_{p}\left(\Delta \phi_{1} ; \phi_{2}\right)
$$

But since

$$
\mathrm{IpC} \vdash\left(E_{p}\left(\Delta \phi_{1}\right) \rightarrow A_{p}\left(\Delta \phi_{1} ; \phi_{2}\right)\right) \Rightarrow A_{p}(\Delta ; \phi)
$$

holds by case (A13) of Table 5 , we get $\operatorname{IpC} \vdash \Gamma \Rightarrow A_{p}(\Delta ; \phi)$, and so in particular (b) holds for $\Gamma \Delta \Rightarrow \phi$.

Case $\left(\rightarrow \Rightarrow_{1}\right)$ : For this rule there are four subcases to consider according to how the active formula and its atomic antecedent occur in $\Gamma \Delta$.

Subcase: $\Gamma=\Gamma^{\prime} q(q \rightarrow \gamma)$ with $q \neq p$ (because $\left.p \notin \operatorname{Var}(\Gamma)\right), \operatorname{IpC} \vdash \Gamma^{\prime} q \gamma \Delta \Rightarrow$ $\phi$, and (a) and (b) hold for this sequent by induction hypothesis. Consequently (a) and (b) hold for $\Gamma \Delta \Rightarrow \phi$ as well, by an application of $\left(\rightarrow_{1}\right)$.

Subcase: $\Gamma=\Gamma^{\prime} q, \Delta=\Delta^{\prime}(q \rightarrow \delta)$, IpC $\vdash \Gamma^{\prime} q \Delta^{\prime} \delta \Rightarrow \phi$, and (a) and (b) hold for this sequent by induction hypothesis. 
(a) If $p \notin \operatorname{Var}(\phi)$, then by (a) for $\Gamma^{\prime} q \Delta^{\prime} \delta \Rightarrow \phi$ we have

$$
\mathrm{IpC} \vdash \Gamma^{\prime} q E_{p}\left(\Delta^{\prime} \delta\right) \Rightarrow \phi
$$

and hence

$$
\mathrm{IpC} \vdash \Gamma\left(q \rightarrow E_{p}\left(\Delta^{\prime} \delta\right)\right) \Rightarrow \phi
$$

by an application of $\left(\rightarrow \Rightarrow_{1}\right)$. But by case (E4) of Table 5 we also have

$$
\mathrm{IpC} \vdash E_{p}(\Delta) \Rightarrow q \rightarrow E_{p}\left(\Delta^{\prime} \delta\right)
$$

and hence (a) holds for $\mathrm{\Gamma} \Delta \Rightarrow \phi$.

(b) By (b) for $\Gamma^{\prime} q \Delta^{\prime} \delta \Rightarrow \phi$ we have

$$
\mathrm{IpC} \vdash \Gamma^{\prime} q E_{p}\left(\Delta^{\prime} \delta\right) \Rightarrow A_{p}\left(\Delta^{\prime} \delta ; \phi\right)
$$

and hence

$$
\mathrm{IpC} \vdash \Gamma\left(q \rightarrow E_{p}\left(\Delta^{\prime} \delta\right)\right) \Rightarrow q \wedge A_{p}\left(\Delta^{\prime} \delta ; \phi\right)
$$

But by cases (E4) and (A4) of Table 5, we also have

$$
\begin{aligned}
& \mathrm{IpC} \vdash E_{p}(\Delta) \Rightarrow q \rightarrow E_{p}\left(\Delta^{\prime} \delta\right) \\
& \mathrm{IpC} \vdash q \wedge A_{p}\left(\Delta^{\prime} \delta ; \phi\right) \Rightarrow A_{p}(\Delta ; \phi)
\end{aligned}
$$

and hence (b) holds for $\Gamma \Delta \Rightarrow \phi$.

Subcase: $\Gamma=\Gamma^{\prime}(q \rightarrow \gamma), \Delta=\Delta^{\prime} q$, IpC $\vdash \Gamma^{\prime} q \gamma \Delta^{\prime} \Rightarrow \phi$, and (a) and (b) hold for this sequent by induction hypothesis.

(a) If $p \notin \operatorname{Var}(\phi)$ then by (a) for $\Gamma^{\prime} q \gamma \Delta^{\prime} \Rightarrow \phi$ we have

$$
\mathrm{IpC} \vdash \Gamma^{\prime} q \gamma E_{p}\left(\Delta^{\prime}\right) \Rightarrow \phi
$$

and hence

$$
\mathrm{IpC} \vdash \Gamma\left(q \wedge E_{p}\left(\Delta^{\prime}\right)\right) \Rightarrow \phi
$$

But by case (E1) of Table 5 we have

$$
\mathrm{IpC} \vdash E_{p}(\Delta) \Rightarrow q \wedge E_{p}\left(\Delta^{\prime}\right)
$$

and hence (a) holds for $\Gamma \Delta \Rightarrow \phi$.

(b) By (b) for $\Gamma^{\prime} q \gamma \Delta^{\prime} \Rightarrow \phi$ we have

$$
\mathrm{IpC} \vdash \Gamma^{\prime} q \gamma E_{p}\left(\Delta^{\prime}\right) \Rightarrow A_{p}\left(\Delta^{\prime} ; \phi\right)
$$

and hence

$$
\vdash \Gamma\left(q \wedge E_{p}\left(\Delta^{\prime}\right)\right) \Rightarrow A_{p}\left(\Delta^{\prime} ; \phi\right)
$$

But by cases (E1) and (A1) of Table 5, we have

$$
\begin{aligned}
& \mathrm{IpC} \vdash E_{p}(\Delta) \Rightarrow q \wedge E_{p}\left(\Delta^{\prime}\right) \\
& \mathrm{IpC} \vdash A_{p}\left(\Delta^{\prime} ; \phi\right) \Rightarrow A_{p}(\Delta ; \phi)
\end{aligned}
$$

and hence (b) holds for $\Gamma \Delta \Rightarrow \phi$. 
Subcase: $\Delta=\Delta^{\prime} d(d \rightarrow \delta)$ with $d$ a propositional variable, IpC $\vdash \Gamma \Delta^{\prime} d \delta \Rightarrow \phi$ and (a) and (b) hold for this sequent by induction hypothesis.

Subsubcase $d=p$ :

(a) If $p \notin \operatorname{Var}(\phi)$, then by (a) for $\Gamma \Delta^{\prime} p \delta \Rightarrow \phi$ we have

$$
\mathrm{IpC} \vdash \Gamma E_{p}\left(\Delta^{\prime} p \delta\right) \Rightarrow \phi
$$

But by case (E5) of Table 5 we also have

$$
\mathrm{IpC} \vdash E_{p}(\Delta) \Rightarrow E_{p}\left(\Delta^{\prime} p \delta\right)
$$

and hence (a) holds for $\Gamma \Delta \Rightarrow \phi$.

(b) By (b) for $\Gamma \Delta^{\prime} p \delta \Rightarrow \phi$ we have

$$
\mathrm{IpC} \vdash \Gamma E_{p}\left(\Delta^{\prime} p \delta\right) \Rightarrow A_{p}\left(\Delta^{\prime} p \delta ; \phi\right)
$$

Hence using cases (E5) and (A5) of Table 5 we get (a) for $\Gamma \Delta \Rightarrow \phi$.

\section{Subsubcase $d \neq p$ :}

(a) If $p \notin \operatorname{Var}(\phi)$, then by (a) for $\Gamma \Delta^{\prime} d \delta \Rightarrow \phi$ we have

$$
\mathrm{IpC} \vdash \Gamma d E_{p}\left(\Delta^{\prime} \delta\right) \Rightarrow \phi
$$

But by cases (E1) and (E4) of Table 5 we have

$$
\begin{aligned}
\mathrm{IpC} \vdash E_{p}(\Delta) & \Rightarrow E_{p}\left(\Delta^{\prime}(d \rightarrow \delta)\right) \wedge d \\
& \Rightarrow\left(d \rightarrow E_{p}\left(\Delta^{\prime} \delta\right)\right) \wedge d \\
& \Rightarrow E_{p}\left(\Delta^{\prime} \delta\right) \wedge d
\end{aligned}
$$

and hence (a) holds for $\Gamma \Delta \Rightarrow \phi$.

(b) By (b) for $\Gamma \Delta^{\prime} d \delta \Rightarrow \phi$ we have

$$
\mathrm{IpC} \vdash \Gamma d E_{p}\left(\Delta^{\prime} \delta\right) \Rightarrow A_{p}\left(\Delta^{\prime} \delta ; \phi\right)
$$

and hence

$$
\mathrm{IpC} \vdash \Gamma E_{p}\left(\Delta^{\prime} \delta\right) \wedge d \Rightarrow d \wedge A_{p}\left(\Delta^{\prime} \delta ; \phi\right)
$$

But as above, by cases (E1) and (E4) of Table 5 we have IpC $\vdash$ $E_{p}(\Delta) \Rightarrow E_{p}\left(\Delta^{\prime} \delta\right) \wedge d$; and similarly, by cases (A4) and (A1) we have

$$
\begin{aligned}
\mathrm{IpC} \vdash d \wedge A_{p}\left(\Delta^{\prime} \delta ; \phi\right) & \Rightarrow A_{p}\left(\Delta^{\prime}(d \rightarrow \delta) ; \phi\right) \\
& \Rightarrow A_{p}\left(\Delta^{\prime} d(d \rightarrow \delta) ; \phi\right)
\end{aligned}
$$

Hence (b) holds for $\Gamma \Delta \Rightarrow \phi$. 
Case $\left(\rightarrow \Rightarrow_{2}\right)$ : This case is analogous to that for $(\wedge \Rightarrow)$, using cases (E6) and (A6) of Table 5 .

Case $\left(\rightarrow \Rightarrow_{3}\right)$ : This case is analogous to that for $(\wedge \Rightarrow)$, using cases (E7) and (A7) of Table 5.

Case $\left(\rightarrow \Rightarrow_{4}\right)$ :

Subcase: $\Gamma=\Gamma^{\prime}\left(\left(\gamma_{1} \rightarrow \gamma_{2}\right) \rightarrow \gamma_{3}\right)$, IpC $\vdash \Gamma^{\prime}\left(\gamma_{2} \rightarrow \gamma_{3}\right) \Delta \Rightarrow \gamma_{1} \rightarrow \gamma_{2}$, IpC $\vdash$ $\Gamma^{\prime} \gamma_{3} \Delta \Rightarrow \phi$ and (a) and (b) hold for these sequents by induction hypothesis, i.e.

$$
\begin{aligned}
& \mathrm{IpC} \vdash \Gamma^{\prime}\left(\gamma_{2} \rightarrow \gamma_{3}\right) E_{p}(\Delta) \Rightarrow \gamma_{1} \rightarrow \gamma_{2} \\
& \mathrm{IpC} \vdash \Gamma^{\prime} \gamma_{3} E_{p}(\Delta) \Rightarrow A_{p}(\Delta ; \phi)
\end{aligned}
$$

and

$$
\mathrm{IpC} \vdash \Gamma^{\prime} \gamma_{3} E_{p}(\Delta) \Rightarrow \phi
$$

when $p \notin \operatorname{Var}(\phi)$. Then (a) and (b) for $\Gamma \Delta \Rightarrow \phi$ follow from these by an application of $\left(\rightarrow \Rightarrow_{4}\right)$.

Subcase: $\Delta=\Delta^{\prime}\left(\left(\delta_{1} \rightarrow \delta_{2}\right) \rightarrow \delta_{3}\right)$, IpC $\vdash \Gamma \Delta^{\prime}\left(\delta_{2} \rightarrow \delta_{3}\right) \Rightarrow \delta_{1} \rightarrow \delta_{2}$, IpC $\vdash$ $\Gamma \Delta^{\prime} \delta_{3} \Rightarrow \phi$ and (a) and (b) hold for these sequents by induction hypothesis, i.e.

$$
\begin{aligned}
& \mathrm{IpC} \vdash \Gamma E_{p}\left(\Delta^{\prime}\left(\delta_{2} \rightarrow \delta_{3}\right)\right) \Rightarrow A_{p}\left(\Delta^{\prime}\left(\delta_{2} \rightarrow \delta_{3}\right) ; \delta_{1} \rightarrow \delta_{2}\right) \\
& \mathrm{IpC} \vdash \Gamma E_{p}\left(\Delta^{\prime} \delta_{3}\right) \Rightarrow A_{p}\left(\Delta^{\prime} \delta_{3} ; \phi\right)
\end{aligned}
$$

and

$$
\mathrm{IpC} \vdash \Gamma E_{p}\left(\Delta^{\prime} \delta_{3}\right) \Rightarrow \phi
$$

when $p \notin \operatorname{Var}(\phi)$ - in which case combining (2), (4) and case (E8) of Table 5 we have that (a) holds for $\Gamma \Delta \Rightarrow \phi$. For (b), from (2) we get

$$
\mathrm{IpC} \vdash \mathrm{T} \Rightarrow E_{p}\left(\Delta^{\prime}\left(\delta_{2} \rightarrow \delta_{3}\right)\right) \rightarrow A_{p}\left(\Delta^{\prime}\left(\delta_{2} \rightarrow \delta_{3}\right) ; \delta_{1} \rightarrow \delta_{2}\right)
$$

and this together with (3) and case (E8) of Table 5 yield

$$
\mathrm{IpC} \vdash \Gamma E_{p}(\Delta) \Rightarrow A_{p}\left(\Delta^{\prime} \delta_{3} ; \phi\right)
$$

Then (5), (6) and case (A8) of Table 5 together give that (b) holds for $\Gamma \Delta \Rightarrow \phi$.

This completes the proof of Proposition 5. 


\section{Interpreting $\mathrm{IpC}^{2}$ in $\mathrm{IpC}$}

Using the propositions $A_{p} \phi$ defined in the previous section, we can translate second order propositions into first order ones.

Definition 7 For each second order proposition $\phi$, define a first order proposition, $\phi^{*}$, by induction on the structure of $\phi$ as follows:

$$
\begin{aligned}
p^{*} & \stackrel{\text { def }}{=} p \\
\perp^{*} & \stackrel{\text { def }}{=} \perp \\
(\phi \# \psi)^{*} & \stackrel{\text { def }}{=} \phi^{*} \# \psi^{*} \quad(\#=\wedge, \vee, \rightarrow) \\
(\forall p \phi) & \stackrel{\text { def }}{=} A_{p} \phi^{*}
\end{aligned}
$$

In order to see that this translation sends $\mathrm{IpC}^{2}$-provable sequents to IpCprovable ones, we need to establish a crucial property of the mapping $\phi \mapsto A_{p} \phi$, namely that it commutes with substitution. It is a peculiarity of second order logic (compared with third, or higher, order logic) that this follows automatically from the properties of $A_{p} \phi$ established in Theorem 1

Lemma 8 Given distinct propositional variables $p, q$, and first order propositions $\phi$ and $\psi$ with $p, q \notin \operatorname{Var}(\psi)$, one has

$$
\mathrm{IpC} \vdash \emptyset \Rightarrow A_{p}(\phi[\psi / q]) \leftrightarrow\left(A_{p} \phi\right)[\psi / q]
$$

Proof By part (ii) of Theorem 1 we have IpC $\vdash A_{p} \phi \Rightarrow \phi$ and hence IpC $\vdash$ $\left(A_{p} \phi\right)[\psi / q] \Rightarrow \phi[\psi / q]$; but since $p$ does not occur in $\left(A_{p} \phi\right)[\psi / q]$, we can apply part (i) of the theorem to conclude that $\operatorname{IpC} \vdash\left(A_{p} \phi\right)[\psi / q] \Rightarrow A_{p}(\phi[\psi / q])$.

To prove the converse, we use the following congruence property of $\leftrightarrow$ in IpC:

$$
\mathrm{IpC} \vdash\left(\psi \leftrightarrow \psi^{\prime}\right) \Rightarrow \theta[\psi / q] \leftrightarrow \theta\left[\psi^{\prime} / q\right]
$$

From part (ii) of Theorem 1 we have

$$
\mathrm{IpC} \vdash A_{p}(\phi[\psi / q]) \Rightarrow \phi[\psi / q]
$$

and so using (7), we also have

$$
\operatorname{IpC} \vdash(\psi \leftrightarrow q) A_{p}(\phi[\psi / q]) \Rightarrow \phi
$$

Since $p$ does not occur in the left-hand side of this sequent, part (i) of the theorem implies that we also have

$$
\mathrm{IpC} \vdash(\psi \leftrightarrow q) A_{p}(\phi[\psi / q]) \Rightarrow A_{p} \phi
$$

On substituting $\psi$ for $q$ throughout this sequent, we obtain

$$
\mathrm{IpC} \vdash A_{p}(\phi[\psi / q]) \Rightarrow\left(A_{p} \phi\right)[\psi / q]
$$

as required. 
Proposition 9 The translation $\phi \mapsto \phi^{*}$ has the following properties:

(i) For all sequents of second order propositions, if $\mathrm{IpC}^{2} \vdash \Gamma \Rightarrow \phi$, then $\mathrm{IpC} \vdash$ $\Gamma^{*} \Rightarrow \phi^{*}$ (where $\Gamma^{*}$ indicates the translation applied elementwise to the multiset $\Gamma)$.

(ii) If $\phi$ is a first order proposition then $\phi^{*}=\phi$.

Thus (-) gives an interpretation of $\mathrm{IpC}^{2}$ into $\mathrm{IpC}$ which restricts to the identity on first order propositions.

Proof Part (i) is proved by induction on the structure of the proof of $\Gamma \Rightarrow \phi$ from the rules in Tables 1 and 2. The induction step for rule ( $\forall$ Intr) uses part (i) of Theorem 1. The induction step for rule $(\forall$ Elim) uses part (ii) of the theorem in conjunction with Lemma 8.

Part (ii) is immediate from the definition of $\left({ }_{-}\right)^{*}$.

Since IpC-provability is decidable whereas IpC $\mathrm{C}^{2}$-provability is not (see [5], for example), the interpretation (_)* cannot be conservative. Here is an interesting example of an unprovable second order proposition whose interpretation is provable.

Example 10 For all second order propositions $\phi$ and $\psi$

$$
\mathrm{IpC} \vdash \emptyset \Rightarrow[\forall p(\phi \vee \psi) \leftrightarrow(\forall p \phi \vee \forall p \psi)]^{*}
$$

For combining Definition 7 with the definition of $A_{p}$ in (1), we have

$$
(\forall p(\phi \vee \psi))^{*}=A_{p}\left(\emptyset ; \phi^{*} \vee \psi^{*}\right)
$$

The construction of $A_{p}$ according to Table 5 then gives

$$
\begin{aligned}
A_{p}\left(\emptyset ; \phi^{*} \vee \psi^{*}\right) & =A_{p}\left(\emptyset ; \phi^{*}\right) \vee A_{p}\left(\emptyset ; \psi^{*}\right) \\
& =(\forall p \phi)^{*} \vee(\forall p \psi)^{*}
\end{aligned}
$$

Second order intuitionistic propositional logic has an interpolation property which is a trivial consequence of quantification over propositions. Using the interpretation (-) ${ }^{*}$ we can transfer this to a non-trivial interpolation property for $\mathrm{IpC}$ which strengthens the usual Interpolation Theorem for this logic.

Proposition 11 Let $\phi$ and $\psi$ be first order propositions for which $\mathrm{IpC} \vdash \phi \Rightarrow \psi$ holds. Call a first order proposition $\theta$ an interpolant for $(\phi, \psi)$ if

- $\operatorname{Var}(\theta) \subseteq \operatorname{Var}(\phi) \cap \operatorname{Var}(\psi)$

- $\mathrm{IpC} \vdash \phi \Rightarrow \theta$ and $\mathrm{IpC} \vdash \theta \Rightarrow \psi$ 
There exist first order propositions $\varepsilon$ and $\alpha$ which are respectively least and greatest interpolants for $(\phi, \psi)$, in the sense that any interpolant $\theta$ satisfies

$$
\mathrm{IpC} \vdash \varepsilon \Rightarrow \theta \quad \text { and } \quad \mathrm{IpC} \vdash \theta \Rightarrow \alpha
$$

Proof Suppose that

$$
\begin{aligned}
\operatorname{Var}(\phi) & =\left\{p_{1}, \ldots, p_{u}, q_{1}, \ldots, q_{v}\right\} \\
\operatorname{Var}(\psi) & =\left\{q_{1}, \ldots, q_{v}, r_{1}, \ldots, r_{w}\right\}
\end{aligned}
$$

with the variables $p_{i}, q_{j}, r_{k}$ pairwise distinct. Since $\mathrm{IpC} \vdash \phi \Rightarrow \psi$, it is also the case that $\mathrm{IpC}^{2} \vdash \phi \Rightarrow \psi$ and hence

$$
\begin{aligned}
& \mathrm{IpC}^{2} \quad \vdash \quad \exists p_{1} \cdots \exists p_{u} \phi \Rightarrow \psi \\
& \mathrm{IpC}^{2} \vdash \quad \phi \Rightarrow \forall r_{1} \cdots \forall r_{w} \psi
\end{aligned}
$$

since $p_{1}, \ldots, p_{u} \notin \operatorname{Var}(\psi)$ and $r_{1}, \ldots, r_{w} \notin \operatorname{Var}(\phi)$. Furthermore, one always has

$$
\begin{aligned}
& \mathrm{IpC}^{2} \vdash \phi \Rightarrow \exists p_{1} \cdots \exists p_{u} \phi \\
& \mathrm{IpC}^{2} \vdash \forall r_{1} \cdots \forall r_{w} \psi \Rightarrow \psi
\end{aligned}
$$

So setting

$$
\begin{aligned}
& \varepsilon \stackrel{\text { def }}{=}\left(\exists p_{1} \cdots \exists p_{u} \phi\right)^{*} \\
& \alpha \stackrel{\text { def }}{=}\left(\forall r_{1} \cdots \forall r_{w} \psi\right)^{*}
\end{aligned}
$$

properties (i) and (ii) in Proposition 9 applied to the above sequents imply that $\varepsilon$ and $\alpha$ are indeed interpolants for $(\phi, \psi)$ in IpC. Moreover, if $\theta$ is any other first order interpolant, from IpC $\vdash \theta \Rightarrow \psi$ we get $\mathrm{IpC}^{2} \vdash \theta \Rightarrow \psi$ and hence $\mathrm{IpC}^{2} \vdash \theta \Rightarrow \forall r_{1} \cdots \forall r_{w} \psi$ ( since $r_{1}, \ldots, r_{w} \notin \operatorname{Var}(\theta)$ ); and then on applying (-)* we get $\mathrm{IpC} \vdash \theta \Rightarrow \alpha$. Similarly, IpC $\vdash \phi \Rightarrow \theta$ implies IpC $\vdash \varepsilon \Rightarrow \theta$.

\section{$4 \quad$ Heyting Algebra Applications}

Recall that a Heyting algebra, $H$, is a (distributive) lattice in which every pair of elements $h, h^{\prime} \in H$ possesses a relative pseudocomplement, $h \rightarrow h^{\prime}$ (the greatest element whose meet with $h$ lies underneath $h^{\prime}$ in the ordering on $H$ ). A morphism of Heyting algebras is a function preserving all finite meets, finite joins and relative pseudocomplements. We will denote by Heyt the category of Heyting algebras and morphisms. As their name suggests, Heyting algebras are the models of an algebraic theory: see for example Balbes and Dwinger [1, Chapter IX] for 
an equational presentation and further information on the theory of Heyting algebras.

The relationship between Heyting algebras and intuitionistic logic is exactly analagous to that between Boolean algebras and classical logic. In particular, there is a correspondence between Heyting algebras and first order intuitionistic propositional theories, induced by the process of forming the Lindenbaum algebra of a theory. Thus for each set $G$, let $\mathrm{F}\langle G\rangle$ denote the set of first order propositions built up from the elements of $G$ regarded as propositional variables. Given a subset $R \subseteq \mathrm{F}\langle G\rangle$, let

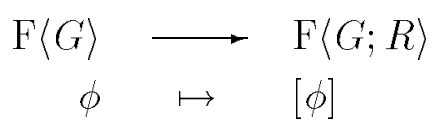

denote the quotient of $\mathrm{F}\langle G\rangle$ by the equivalence relation identifying two first order propositions $\phi$ and $\psi$ if and only if $\mathrm{IpC} \vdash \Gamma \Rightarrow \phi \leftrightarrow \psi$ holds for some finite $\Gamma \subseteq R$. Endowing $\mathrm{F}\langle G ; R\rangle$ with the partial order

$$
[\phi] \leq[\psi] \text { if and only if for some finite } \Gamma \subseteq R, \quad \operatorname{IpC} \vdash \Gamma \Rightarrow \phi \rightarrow \psi
$$

one obtains a Heyting algebra - the Lindenbaum algebra of the IpC-theory (determined by) $R$ over the language $G$. Moreover, every Heyting algebra, $H$, can be presented in this way: for example, take $G$ to be the set $H$ itself and $R$ to be those propositions mapped to $\top$ by the obvious evaluation function $\mathrm{F}\langle G\rangle \rightarrow H$ induced by the identity function $G \rightarrow H$.

Theorem 1 can be rephrased as a statement about the relationship between a Heyting algebra $H$ and the algebra obtained from it by freely adjoining an indeterminate $X$.

Definition 12 Given a Heyting algebra $H$, a Heyting polynomial algebra over $H$ is a Heyting algebra $H[X]$ equipped with a distinguished element $X$ and a morphism $i_{H}: H \longrightarrow H[X]$ with the following universal property:

For each morphism $f: H \longrightarrow K$ in Heyt and each element $k \in K$, there is a unique morphism $g: H[X] \longrightarrow K$ such that $g \circ i_{H}=f$ and $g(X)=k$.

As usual, the universal property in the definition determines $H[X]$ uniquely up to isomorphism (over $H$ ). Given a presentation of $H$ as $\mathrm{F}\langle G ; R\rangle$, one can present $H[X]$ as $\mathrm{F}\langle G \cup\{p\} ; R\rangle$, where $p$ is any element not contained in $G$; the morphism $i_{H}$ is induced by the inclusion $\mathrm{F}\langle G\rangle \subseteq \mathrm{F},\langle G \cup\{p\}\rangle$, and the distinguished element $X$ is the equivalence class $[p]$.

When $R=\emptyset, H=\mathrm{F}\langle G ; R\rangle$ is the free Heyting algebra on the set of generators $G$ and $H[X]$ is free on $G \cup\{p\}$. Except for the case of a single generator, the structure of free Heyting algebras is not well understood. The following theorem sheds some new light on this structure. 
Theorem 13 For any Heyting algebra $H$, the morphism $i_{H}: H \longrightarrow H[X]$ possesses both left and right adjoints. In other words there exist functions

$$
e_{H}: H[X] \longrightarrow H \quad \text { and } \quad a_{H}: H[X] \longrightarrow H
$$

satisfying

$$
\begin{array}{ll}
e_{H}(P) \leq h & \text { if and only if } P \leq i_{H}(h) \\
h \leq a_{H}(P) & \text { if and only if } \quad i_{H}(h) \leq P
\end{array}
$$

for all $P \in H[X]$ and $h \in H$.

These adjoints are natural in $H$. In other words, for each morphism $f$ : $H \longrightarrow H^{\prime}$ in Heyt the following squares commute
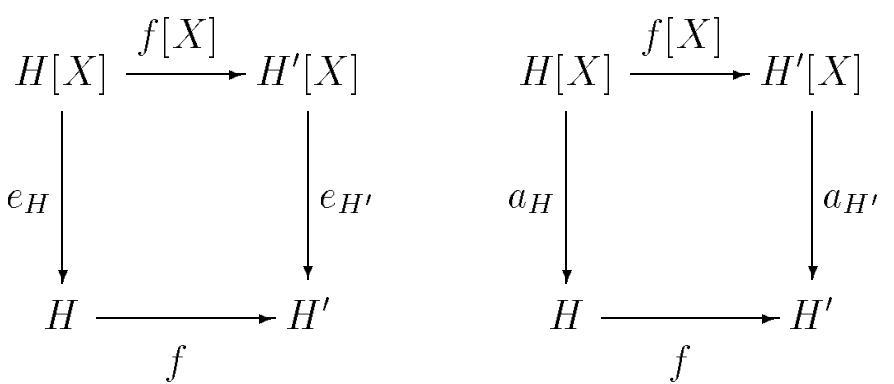

(where $f[X]$ is the unique morphism g satisfying $\left(g \circ i_{H}\right)=\left(i_{H^{\prime}} \circ f\right)$ and $g(X)=X$ whose existence is guaranteed by the universal property of $H[X]$ in Definition 12).

Proof As remarked above we can assume $H=\mathrm{F}\langle G ; R\rangle$ for some $G$ and $R$, and then take $H[X]=\mathrm{F}\langle G \cup\{p\} ; R\rangle$. Thus each element $h \in H$ is of the form $h=[\psi]$, where $\psi$ is some first order proposition with variables in $G$. Similarly, each $P \in H[X]$ is of the form $P=[\phi]$ where $\phi$ may involve $p$ as well variables in $G$. Moreover, $i_{H}(h) \leq P$ in $H[X]$ if and only if $\mathrm{IpC} \vdash \Gamma \Rightarrow \psi \rightarrow \phi$ holds for some finite $\Gamma \subseteq R$. But by Theorem 1 we have

$$
\begin{array}{rll}
\mathrm{IpC} \vdash \Gamma \Rightarrow \psi \rightarrow \phi & \text { iff } & \mathrm{IpC} \vdash \mathrm{T} \psi \Rightarrow \phi \\
& \text { iff } & \mathrm{IpC} \vdash \Gamma \psi \Rightarrow A_{p} \phi \\
& \text { iff } & \mathrm{IpC} \vdash \Gamma \Rightarrow \psi \rightarrow A_{p} \phi
\end{array}
$$

So defining $a_{H}(P)=\left[A_{p} \phi\right]$ we get $(9)$. (Clearly the definition of $a_{H}(P)$ is independent of the choice of representative for $P$.) The naturality of $a_{H}$ follows from Lemma 8, because any morphism $\mathrm{F}\langle G ; R\rangle \longrightarrow \mathrm{F}\left\langle G^{\prime} ; R^{\prime}\right\rangle$ is induced by a function $\mathrm{F}\langle G\rangle \longrightarrow \mathrm{F}\left\langle G^{\prime}\right\rangle$ of the form

$$
\psi \mapsto \psi\left[\gamma_{g}^{\prime} / g \mid g \in G\right]
$$

for some $G$-indexed family $\left(\gamma_{g}^{\prime} \in \mathrm{F}\left\langle G^{\prime}\right\rangle \mid g \in G\right)$. Alternatively, the naturality of $a_{H}$ can be deduced from an interpolation property of pushout squares in Heytsee $[9$, Theorem B]. 
The existence of the left adjoints $e_{H}$ (and their naturality), follows from the existence of the natural right adjoints by an algebraic version of the proof that $\exists$ is definable from $\forall$ and $\rightarrow$ in IpC ${ }^{2}$. Alternatively, we can use Proposition 5 to give a direct definition:

$$
e_{H}([\phi])=\left[E_{p}(\phi)\right]
$$

Remarks 14 (i) The morphism $i_{H}: H \longrightarrow H[X]$ is always a monomorphism (since the universal property of $H[X]$ implies that $i_{H}$ has a left inverse sending $X$ to, for example, the top element of $H$ ). Consequently the adjoints to $i_{H}$ satisfy

$$
e_{H} \circ i_{H}=i d_{H}=a_{H} \circ i_{H}
$$

(Of course they also satisfy $i_{H} \circ a_{H} \leq i d_{H[X]} \leq i_{H} \circ e_{H}$.)

(ii) (Cf. Remark 2.) The analogue of Theorem 13 for Boolean algebras is rather trivial. This is because a Boolean polynomial in a single indeterminate $X$ with coefficients in a given Boolean algebra $B$, can always be put in the normal form

$$
(b \wedge X) \vee\left(b^{\prime} \wedge \neg X\right) \quad\left(b, b^{\prime} \in B\right)
$$

Then the inclusion of $B$ into its algebra of Boolean polynomials is given by

$$
b \mapsto(b \wedge X) \vee(b \wedge \neg X)
$$

and it is easy to see that this has left and right adjoints, given respectively by $(b \wedge X) \vee\left(b^{\prime} \wedge \neg X\right) \mapsto\left(b \vee b^{\prime}\right)$ and $(b \wedge X) \vee\left(b^{\prime} \wedge \neg X\right) \mapsto\left(b \wedge b^{\prime}\right)$.

Consider the morphism

$$
k: \mathrm{F}\langle G ; R\rangle \longrightarrow \mathrm{F}\langle G \cup\{p\} ; R \cup\{\rho\}\rangle
$$

induced by the inclusion $\mathrm{F}\langle G\rangle \subseteq \mathrm{F}\langle G \cup\{p\}\rangle$, where as before $p$ is some new element not in $G$ and now we have also extended $R$ by adding in some $\rho \in$ $\mathrm{F}\langle G \cup\{p\}\rangle$. The proof of Theorem 13 extends to show that this morphism $k$ also has left and right adjoints, $e_{k}, a_{k}$, given by:

$$
\begin{aligned}
e_{k}([\phi]) & =\left[E_{p}(\rho \wedge \phi)\right] \\
a_{k}([\phi]) & =\left[A_{p}(\rho \rightarrow \phi)\right]
\end{aligned}
$$

Iterating, we get adjoints for the morphism induced by extending $G$ and $R$ by finitely many elements. Such morphisms are precisely the finitely presented objects in the locally finitely presentable category $H /$ Heyt of Heyting algebras equipped with a morphism from $H(=\mathrm{F}\langle G ; R\rangle)$ (and whose morphisms are commutative triangles). So we get the following corollary (the second paragraph of which follows from the first and the interpolation property [9, Theorem B] of pushout squares in $\mathbf{H e y t}$ ): 
Corollary 15 Any morphism $k: H \longrightarrow K$ in Heyt which makes $K$ finitely presented over $H$, possesses both left and right adjoints, $e_{k}, a_{k}: K \longrightarrow H$.

Furthermore, given any morphism $f: H \longrightarrow H^{\prime}$, forming the pushout square

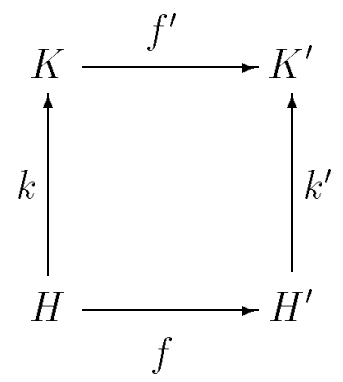

in Heyt, ( $k^{\prime}$ necessarily makes $K^{\prime}$ finitely presented over $H^{\prime}$ and) the adjoints satisfy $f \circ e_{k}=e_{k^{\prime}} \circ f^{\prime}$ and $f \circ a_{k}=a_{k^{\prime}} \circ f^{\prime}$.

These results enables us to construct for each Heyting algebra $H$, a model of the second order calculus $\mathrm{IpC}^{2}$. To explain further, we must describe what is needed to specify such a model. The particular notion of model we will use is the specialization from categories to partial orders of the notion of model of the second order lambda calculus described in $[10,12]$.

First note that since the notion of Heyting algebra is algebraic, it makes sense to speak of a Heyting algebra object, $U$, in any category $\mathcal{C}$ with finite products: such an object comes equipped with morphisms

$$
\top, \perp: 1 \longrightarrow U \quad \wedge, \vee, \rightarrow: U \times U \longrightarrow U
$$

making various diagrams (derived from the defining equations of the theory of Heyting algebras) commute in $\mathcal{C}$. As usual, this structure induces an ordinary Heyting algebra structure on the hom-sets $\mathcal{C}(I, U)$; and precomposition with $f$ : $I \longrightarrow I^{\prime}$ gives a morphism of Heyting algebras, $f^{*}: \mathcal{C}\left(I^{\prime}, U\right) \longrightarrow \mathcal{C}(I, U)$.

Definition 16 Say that a Heyting algebra object $U$ in $\mathcal{C}$ possesses internal $U$ indexed meets if for each object $I \in \mathcal{C}$ there is a right adjoint

$$
\bigwedge_{I}: \mathcal{C}(I \times U, U) \longrightarrow \mathcal{C}(I, U)
$$

to the morphism $\pi_{1}^{*}$ induced by composition with the first projection morphism $\pi_{1}: I \times U \longrightarrow I$, and moreover these right adjoints are natural in $I$.

Remark 17 The import of this condition on $U$ becomes more apparent from the point of view of the internal higher order logic of the topos $\left[\mathcal{C}^{\mathrm{op}}, \mathcal{S}\right.$ et $]$ of presheaves on $\mathcal{C}$. Identifying the objects $I$ of $\mathcal{C}$ with their corresponding presheaves $\mathcal{C}\left({ }_{-}, I\right)$, the functions $\bigwedge_{I}$ constitute a morphism $U^{U} \longrightarrow U$ giving the meet of an internal $U$-indexed family of elements of $U$. 
A Heyting algebra object $U$ possessing internal $U$-indexed meets in a category $\mathcal{C}$ with finite products, determines a model of $\mathrm{IpC}^{2}$. For each second order proposition $\phi$ with free variables in the list $\vec{p}=p_{1}, \ldots, p_{n}$ of distinct variables, we get a morphism

$$
\llbracket \phi(\vec{p}) \rrbracket: U^{n} \longrightarrow U
$$

defined by induction on the structure of $\phi$ as follows:

$$
\begin{aligned}
\llbracket p_{i}(\vec{p}) \rrbracket & \stackrel{\text { def }}{=}\left(U^{n} \stackrel{\pi_{i}}{\longrightarrow} U\right) \\
\llbracket \perp(\vec{p}) \rrbracket & \stackrel{\text { def }}{=}\left(U^{n} \longrightarrow 1 \stackrel{\perp}{\longrightarrow} U\right) \\
\llbracket(\phi \# \psi)(\vec{p}) \rrbracket & \stackrel{\text { def }}{=}\left(U^{n} \stackrel{\langle\llbracket \phi(\vec{p}) \rrbracket \llbracket \llbracket(\vec{p}) \rrbracket\rangle}{\longrightarrow} U \times U \stackrel{\#}{\longrightarrow} U\right) \\
\llbracket(\forall p \phi)(\vec{p}) \rrbracket \stackrel{\text { def }}{=} & \Lambda_{U^{n}}\left(U^{n} \times U \stackrel{\llbracket \phi(\vec{p} p) \rrbracket}{\longrightarrow} U\right)
\end{aligned}
$$

(where $\#=\wedge, \vee$, or $\rightarrow$ ). In particular, when $\phi$ contains no free variables we can take $\vec{p}$ to be empty and obtain a global element

$$
\llbracket \phi \rrbracket \in \mathcal{C}(1, U)
$$

of $U$ for each closed second order proposition $\phi$. We will call the Heyting algebra $\mathcal{C}(1, U)$ the algebra of truth-values of the model $(\mathcal{C}, U)$.

Say that $(\mathcal{C}, U)$ satisfies such a closed proposition $\phi$ and write $(\mathcal{C}, U) \models \phi$ if $\llbracket \phi \rrbracket$ is the top element of $\mathcal{C}(1, U)$. This notion of satisfaction is sound for provability in $\mathrm{IpC}^{2}$ : if $\mathrm{IpC}^{2} \vdash \emptyset \Rightarrow \phi$ then $(\mathcal{C}, U) \models \phi$. Conversely, it is not hard to prove (by a term model construction) that it is also complete: $\mathrm{IpC}^{2} \vdash \emptyset \Rightarrow \phi$ holds if $\phi$ is satisfied by all $(\mathcal{C}, U)$.

Returning now to Theorem 13, given $H \in$ Heyt, let $\mathcal{C}$ be the opposite of the full subcategory of $H$ /Heyt consisting of the finitely generated free objects. More concretely, we can take the objects of $\mathcal{C}$ to be finite ordinals, $[n]$, and the morphisms $[n] \longrightarrow[m]$ to be $m$-tuples of elements of the polynomial Heyting algebra in $n$ indeterminates, $H\left[X_{1}, \ldots, X_{n}\right]$. Composition is given by substitution and the identity on $[n]$ is $\left(X_{1}, \ldots, X_{n}\right)$. Lawvere's categorical treatment of algebraic theories (see [7], for example) tells us that $\mathcal{C}$ (has finite products and) contains the generic model of the algebraic theory of 'Heyting algebras equipped with a morphism from $H^{\prime}$. In particular, $\mathcal{C}$ does contain a Heyting algebra object, namely $U=[1]:$ its top and bottom elements are $(\top),(\perp):[0] \longrightarrow[1]$ and its meet, join and pseudocomplementation operations are $\left(X_{1} \# X_{2}\right):[1] \times[1]=[2] \longrightarrow[1]$ (for $\#=\wedge, \vee, \rightarrow)$.

For this Heyting algebra object we have for each object $[n] \in \mathcal{C}$ that

$$
\begin{aligned}
\mathcal{C}([n], U)=\mathcal{C}([n],[1]) & =H\left[X_{1}, \ldots, X_{n}\right] \\
\mathcal{C}([n] \times U, U)=\mathcal{C}([n+1],[1]) & =H\left[X_{1}, \ldots, X_{n}, X_{n+1}\right] \\
& \cong H\left[X_{1}, \ldots, X_{n}\right][X]
\end{aligned}
$$


and $\pi_{1}^{*}$ is $i_{H\left[X_{1}, \ldots, X_{n}\right]}$. Consequently, Theorem 13 implies that $U$ has internal $U$ indexed meets and hence determines a model of $\mathrm{IpC}^{2}$. Note that the algebra of truth-values of this model is $\mathcal{C}(1, U)=\mathcal{C}([0],[1])=H$, the given Heyting algebra. We have thus proved:

Proposition 18 Given a Heyting algebra $H$, the algebraic theory of 'Heyting algebras equipped with a morphism from $H$ ' has the property that its generic model $U$ has internal $U$-indexed meets, and hence provides a model of second order intuitionistic propositional logic. Since the algebra of truth-values of this model is just $H$, we conclude that every Heyting algebra appears as the algebra of truth-values of some model of $I p C^{2}$.

There is a correspondence between instances of the notion of model of $I p C^{2}$ as we have defined it, and $I p C^{2}$-theories. (By such a theory we mean a suitable language together with a collection of axioms - second order propositions over the language.) Under this correspondence, the algebra of truth-values of a model is identified with the Lindenbaum algebra of the theory (i.e. the collection of closed second order propositions over the given language, quotiented by provability in $I p C^{2}$ augmented with the given axioms). Consequently, Proposition 18 implies:

Every Heyting algebra is the Lindenbaum algebra of some $I p C^{2}$-theory.

In fact one can see this without recourse to the correspondence between models and theories, using the interpretation of $\mathrm{IpC}^{2}$ into $\mathrm{IpC}$ developed in section 3 . For given a Heyting algebra $H$, choose a presentation for it as $H=\mathrm{F}\langle G ; R\rangle$. Then the set of second order propositions over $G$

$$
\left\{\phi \mid \mathrm{IpC} \vdash \Gamma \Rightarrow \phi^{*} \text {, for some finite } \Gamma \subseteq R\right\}
$$

determines an $\mathrm{IpC}^{2}$-theory over $G$ whose Lindenbaum algebra is isomorphic to $\mathrm{F}\langle G ; R\rangle$ (the isomorphism being induced by $\left({ }_{-}\right)^{*}$ ). 


\section{References}

[1] R. Balbes and P. Dwinger, Distributive Lattices (University of Missouri Press, 1974).

[2] N. Dershowitz and Z. Manna, Proving Termination with Multiset Orderings, Communications of the ACM 22(1979) 465-476.

[3] A. G. Dragalin, Mathematical Intuitionism, Transl. Math. Monographs, Vol.69 (Amer. Math. Soc., Providence RI, 1988).

[4] R. Dyckhoff, Contraction-free Sequent Calculi for Intuitionistic Logic, preprint, University of St.Andrews, 1990.

[5] D. M. Gabbay, Semantical Investigations in Heyting's Intuitionistic Logic, Synthese Library Vol. 148 (D. Reidel, Dordrecht, 1981).

[6] J. Hudelmaier, Bounds for Cut Elimination in Intuitionistic Propositional Logic, PhD Thesis, University of Tübingen, 1989.

[7] A. Kock and G. E. Reyes, Doctrines in categorical logic. In: J. Barwise (ed.), Handbook of Mathematical Logic (North-Holland, Amsterdam, 1977), Chapter A.8.

[8] P. Lincoln, A. Scedrov and N. Shankar, Linearizing intuitionistic implication, Proceedings of the 6th Annual Symposium on Logic in Computer Science, Amsterdam, July 1991, IEEE Computer Society Press, Washington, 1991.

[9] A. M. Pitts, Amalgamation and Interpolation in the Category of Heyting Algebras, Jour. Pure Applied Algebra 29(1983) 155-165.

[10] A. M. Pitts, Polymorphism is Set Theoretic, Constructively. In: D. Pitt et al (eds), Category Theory and Computer Science, Proceedings Edinburgh 1987, Lecture Notes in Computer Science Vol. 283 (Springer-Verlag, Berlin, 1987), pp 12-39.

[11] D. Prawitz, Natural Deduction (Almqvist \& Wiksell, Stockholm, 1965).

[12] R. A. G. Seely, Categorical Semantics for Higher Order Polymorphic Lambda Calculus, Jour. Symbolic Logic 52(1987) 969-989.

[13] N. Vorob'ev, A New Algorithm for Derivability in the Constructive Propositional Calculus, Amer. Math. Soc. Transl. (2) 94(1970) 37-71. (Translation from Russian of Trudy Mat. Inst. Steklov 52(1958) 193-225.)

[14] G. R. Renardel de Lavalette, Interpolation in fragments of intuitionistic propositional logic, this JOURNAL, vol. 54 (1989), pp. 1419-1430. 